Revue des patrimoines

\title{
Fiers destriers : images du cheval de guerre au Moyen Âge
}

\section{Marina Viallon}

\section{(2) OpenEdition}

1 Journals

Édition électronique

URL : http://journals.openedition.org/insitu/12066

DOI : 10.4000/insitu. 12066

ISSN : 1630-7305

Éditeur

Ministère de la Culture

Référence électronique

Marina Viallon, «Fiers destriers : images du cheval de guerre au Moyen Âge », In Situ [En ligne], 27 |

2015, mis en ligne le 02 novembre 2015, consulté le 10 décembre 2020. URL : http://

journals.openedition.org/insitu/12066 ; DOI : https://doi.org/10.4000/insitu.12066

Ce document a été généré automatiquement le 10 décembre 2020.

\section{(c) (i) (2)}

In Situ Revues des patrimoines est mis à disposition selon les termes de la licence Creative Commons Attribution - Pas d'Utilisation Commerciale - Pas de Modification 4.0 International. 


\title{
Fiers destriers : images du cheval de guerre au Moyen Âge
}

\author{
Marina Viallon
}

Dans notre imaginaire contemporain du Moyen Âge, la figure du preux chevalier sur sa noble monture occupe une place de choix ${ }^{1}$. Il est en effet indissociable de la chevalerie, cette nouvelle noblesse guerrière qu'ont vu se développer les règnes des premiers Capétiens. Cheval, chevalier, la relation linguistique elle-même est évidente, même si dans les textes latins celui-ci est souvent désigné comme miles, littéralement le soldat. Mais dans la langue vulgaire il est le chevalier (signifiant cavalier en ancien français), le combattant assez riche pour posséder et entretenir son propre cheval. Mais quel était ce cheval ? Contrairement à aujourd'hui, le Moyen Âge ignore les races de chevaux, les animaux étant au mieux désignés par leur origine géographique, mais plus communément en fonction du type d'activité auquel ils étaient destinés. Les chevaux étaient placés dans ces catégories en fonction de leurs aptitudes naturelles et de leur dressage. Ainsi par exemple le palefroi était un cheval de prix dont la capacité à marcher l'amble, allure plus rapide que le pas mais plus confortable pour le cavalier et moins fatigante pour l'animal que le trot, le désignait comme la monture idéale pour la balade et le voyage. Mais le cheval le plus coûteux de l'écurie médiévale était le destrier, l'étalon sélectionné et dressé pour la guerre et le tournoi.

On s'est longtemps interrogé sur l'origine de ce nom, qui, en ancien français, signifie tout simplement droitier. Si beaucoup de linguistes, probablement non cavaliers, ont pu s'accorder avec une explication relative à la main par laquelle étaient tenus ces chevaux (pratique non réservée au seul cheval de guerre d'ailleurs), une autre hypothèse, moins répandue mais pour notre part plus convaincante, renvoie plutôt ce terme à une pratique de dressage spécifique. Le destrier ne serait donc pas le cheval que l'on tient par la main droite, mais plutôt le cheval entraîné à galoper à main droite sur commande, en d'autres termes, un «cheval droitier ». Le galop est en effet une allure asymétrique, et selon l'antérieur qui avance le plus à chaque foulée, le cheval est dit galoper à main droite ou à main gauche, ceci lui permettant naturellement de garder son équilibre dans les courbes. Cette compétence revêt toute son importance dans le développement d'une nouvelle 
technique militaire qui domina les champs de bataille jusqu'à la fin du Moyen Âge : la charge à la lance couchée. En effet, contrairement à ce que l'on pourrait penser, l'arme chevaleresque par excellence n'est pas spécialement l'épée, arme finalement assez commune, mais la lance, arme réservée à la cavalerie. Celle-ci au début ne diffère pas de celle utilisée par les combattants à pied, mais à partir de la fin $\mathrm{du} \mathrm{XI}^{\mathrm{e}}$ siècle, elle commence à être utilisée par les cavaliers d'une manière spécifique qui entraînera pour cette arme une évolution indépendante. Les premières représentations de la technique de la lance couchée apparaissent sur la broderie de Bayeux (fig. 1). Cette technique consiste à maintenir la hampe de la lance fermement serrée contre son côté droit, en faisant passer celle-ci par-dessus le cou du cheval, à gauche, car ce côté était au XI ${ }^{e}$ siècle protégé de l'épaule jusqu'au genou par un grand bouclier. C'est ensuite la force et la vitesse du cheval qui donnent son efficacité à cette attaque, toute la science du cavalier résidant à maintenir une position parfaite permettant à la fois d'asséner le coup et d'encaisser celui de son adversaire sans se faire soi-même désarçonner.

Figure 1

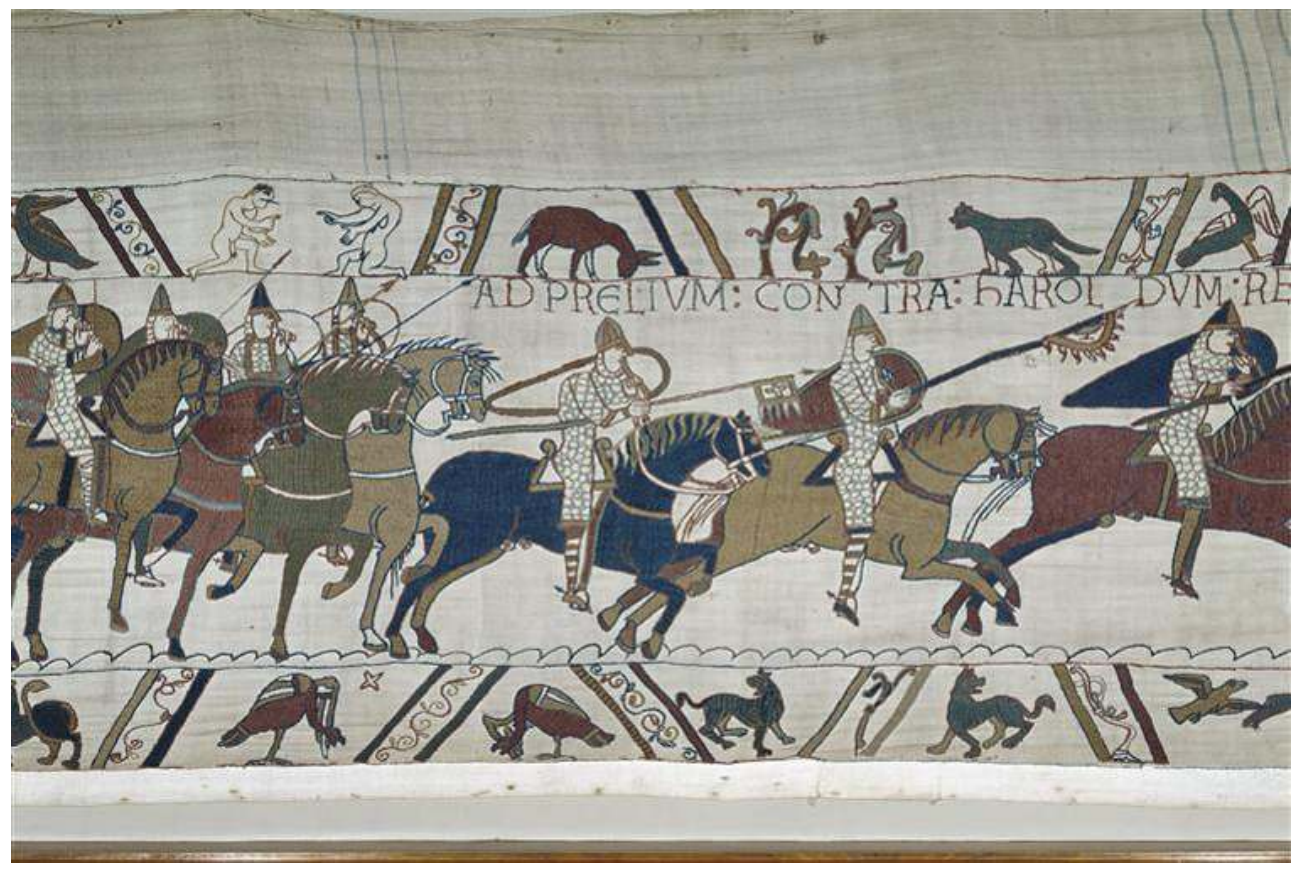

Les cavaliers normands chargent lance couchée à la bataille d'Hastings, broderie de Bayeux, dernier quart du XI siècle. Bayeux, Musée de la Tapisserie.

(c) Ministère de la Culture - Médiathèque du Patrimoine, Dist. RMN-Grand Palais / Jean Gourbeix / Simon Guillot (cote cliché RMN : 13-512230).

3 Ainsi, pour se protéger de la lance ennemie, les chevaliers usant de cette technique faisaient en sorte de se croiser uniquement par leur gauche. Un cheval galopant à main droite s'incurve aussi vers la droite, ce qui réduit notamment les chances que sa tête ne se trouve dans la trajectoire de la lance ennemie. Galoper à main droite permettait également à l'animal de mieux garder son équilibre si son cavalier était frappé par une lance à gauche. Les représentations contemporaines ne peuvent malheureusement pas nous aider à confirmer cette hypothèse, car le galop est toujours représenté dans la position irréaliste du galop volant ou du bond en avant. Dans ces cas-là, les artistes semblent avoir très généralement suivi la convention esthétique qui consiste à avancer l'antérieur se trouvant au second plan de l'image, sans volonté particulière de nous 
montrer un cheval galopant à droite ou à gauche. Cette pratique de dressage est cependant connue et évoquée par les maîtres d'équitation de la Renaissance, comme le Napolitain Federico Grisone, dans son Ordini de Cavalcare (1550), et on peut supposer qu'il couche ici sur papier un savoir centenaire transmis par plusieurs générations d'hommes de cheval ${ }^{2}$. Car l'équitation et le dressage des chevaux au Moyen Âge est un savoir oral, transmis par la pratique et non par l'écrit, et il n'a donc laissé très peu de traces, ce qui a longtemps fait penser qu'il n'existait aucune équitation médiévale. Si elle peut nous paraître moins sophistiquée que celle développée à l'époque moderne, elle répondait également à une utilisation militaire du cheval assez différente. Le destrier devait néanmoins suivre un long entraînement afin qu'il puisse par exemple changer de pied au galop selon les ordres de son cavalier, se familiariser au bruit et à la cohue d'un combat médiéval ou encore courir sans broncher vers un autre cavalier armé d'une lance, attitude bien peu naturelle pour un animal-proie comme le cheval ${ }^{3}$. Les premiers tournois, apparus dans le courant du XII ${ }^{\mathrm{e}}$ siècle, avaient d'ailleurs comme but principal de familiariser hommes et bêtes à cette nouvelle pratique de combat. La pratique de la lance couchée, demandant un très long entraînement depuis l'enfance pour le cavalier comme pour sa monture, était donc par défaut réservée à une élite qui avait le temps et les moyens de s'y consacrer.

Cette pratique eut des conséquences directes sur toute l'évolution de l'armement, du harnachement, et surtout du cheval de guerre. Les animaux étaient sélectionnés pour leur taille, leur agilité et leur force. Ils devaient en effet être assez puissants et charpentés, non pas pour porter le harnois de fer de leurs cavaliers ${ }^{4}$, mais pour pouvoir donner et encaisser les coups de lance, tout en étant suffisamment rapides et maniables pour manœuvrer de manière optimale sur un champ de bataille. Même si jusqu'au XIV ${ }^{\mathrm{e}}$ siècle les représentations souvent très stylisées des chevaux ne nous permettent pas une analyse morphologique très poussée, nous pouvons néanmoins en dégager quelques points. Ceux-ci semblent moyennement graciles, les membres fins, avec souvent une encolure assez forte. Le naturalisme de l'art de la fin du Moyen Âge nous donne des indications plus précises sur ce que sont devenus ces chevaux après quelques siècles de sélection spécialisée. Le cheval de guerre idéal a alors le corps compact et musclé, une forte encolure, des membres fins mais robustes. Ces caractéristiques physiques sont très proches de celles que l'on retrouve aujourd'hui sur les chevaux ibériques par exemple. En effet le cheval dit d'Espagne fut pendant longtemps considéré comme le meilleur cheval de guerre, et il le resta en France jusqu'à la fin du XviII ${ }^{e}$ siècle. On sait que Guillaume le Conquérant, à la bataille d'Hastings, montait un cheval espagnol qui lui avait été offert, bien que rien ne le différencie des autres sur la broderie de Bayeux. Ces animaux, issus de croisements entre des chevaux ibériques endémiques et des barbes importés d'Afrique du Nord par l'occupation arabe, alliaient les qualités à la fois physiques et psychiques idéales pour la guerre, qui sont encore celles revendiquées par le cheval de pure race espagnole. Ces chevaux étaient très chers et leur importation réglementée, et seuls les seigneurs et princes les plus riches montaient de tels animaux, mais des types similaires étaient également recherchés dans des élevages plus locaux. Le destrier se distinguait aussi par sa taille, la plus élevée parmi les chevaux médiévaux, bien que celle-ci semble relativement petite par rapport à nos critères actuels. En effet, celui qui était appelé le "grand cheval » (synonyme alors de destrier) devait alors toiser entre 1,50 $\mathrm{m}$ et 1,60 $\mathrm{m}$ au garrot, ce qui correspond de nos jours aux tailles moyennes des chevaux de pure race espagnole ${ }^{5}$. Ces mesures, corroborées par les études archéozoologiques de cette période ${ }^{6}$, correspondent à 
la plupart des représentations contemporaines, même les plus stylisées. Les cavaliers, qui montaient étriers longs, avaient les pieds arrivant au niveau du ventre de leur monture, voire légèrement au-delà. Et si l'on considère la taille moyenne d'un homme à 1,70 m, ces proportions sont tout à fait correctes pour des chevaux de la taille que nous avons indiquée. Cette différence de taille entre le destrier et les autres chevaux est particulièrement visible sur certaines des illustrations du Livre des tournois du roi René d'Anjou peintes par le peintre Barthélemy d'Eyck (fig. 2), notamment celle illustrant l'entrée des tournoyeurs dans la ville avant le tournoi. Un grand destrier noir, monté comme le préconise René par un "bien petit page ", certainement pour en augmenter visuellement la stature, est suivi par des musiciens ainsi que par le duc de Bretagne et ses chevaliers. Tous sont montés sur des palefrois, bien plus petits; en témoignent les pieds des cavaliers dépassant largement en-dessous du ventre de leurs montures. En effet, on ne montait jamais le destrier pour se rendre au tournoi ou sur le champ de bataille, le but de cette pratique étant de préserver les forces des chevaux pour le combat. De plus, comme nous l'avons dit, un palefroi marchant l'amble était bien plus endurant et confortable pour voyager.

Figure 2

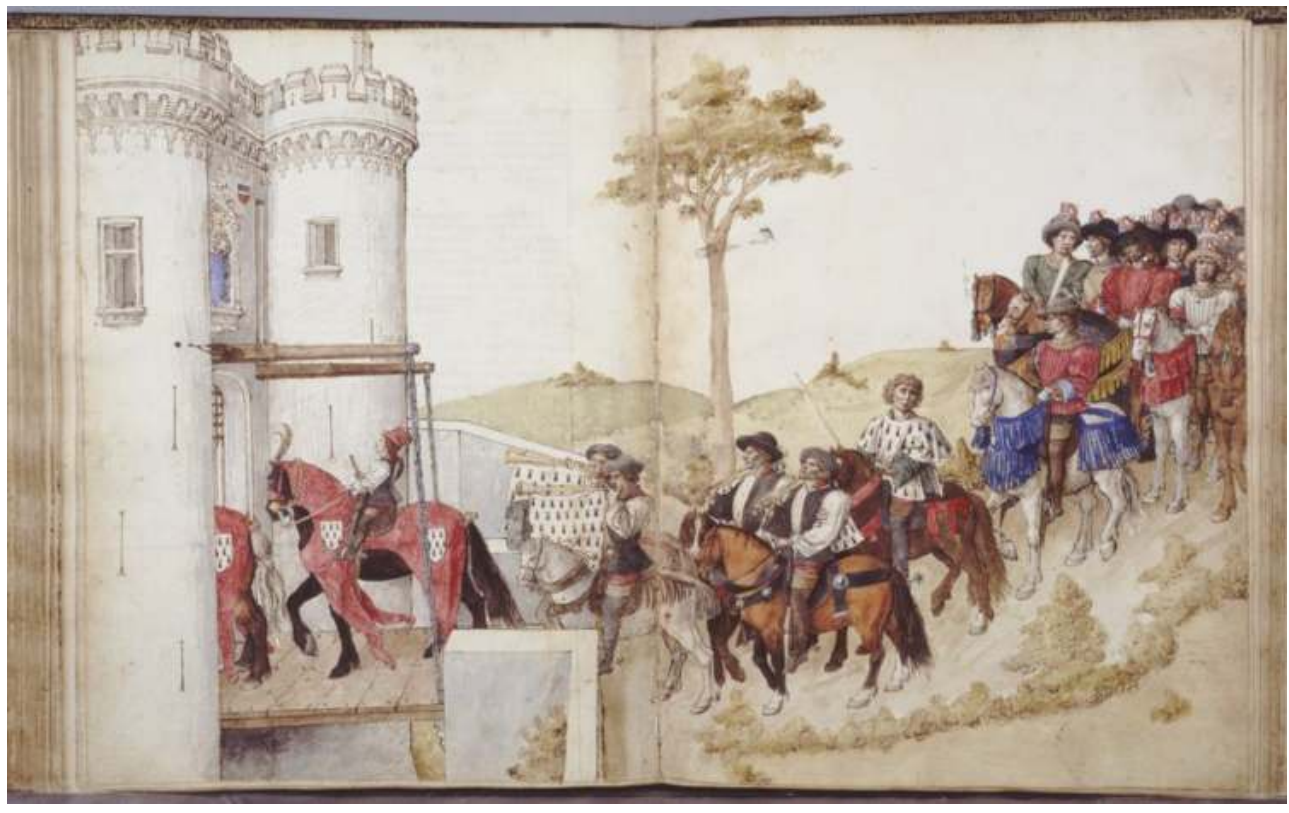

L'arrivée des chevaliers et des destriers dans la ville, René d'Anjou, Le livre des tournois, 1460-1465. Paris, Bibliothèque nationale de France (BnF, ms. fr. 2695, fol. 151v-152).

(C) BnF.

Cette image est l'occasion pour nous d'évoquer un autre aspect important du cheval de guerre médiéval : sa symbolique. Si René met en avant les destriers dans son traité, c'est qu'ils sont devenus, avec l'essor de la chevalerie, un élément fort de l'identité de cette classe guerrière. Un cheval est un animal cher à l'achat et à l'entretien, d'autant plus, que contrairement aux autres gros animaux domestiques, il ne produit ni lait ni laine et la consommation de sa viande est officiellement proscrite par l'Église. Si l'on ajoute la taille exceptionnelle du destrier et son dressage, sa possession est un luxe que l'on est fier d'exhiber en public, que ce soit dans la réalité ou sur les représentations. Une page du 
Psautier de Westminster, datée des environs de 1250, montre un chevalier agenouillé devant un roi (fig. 3).

Figure 3

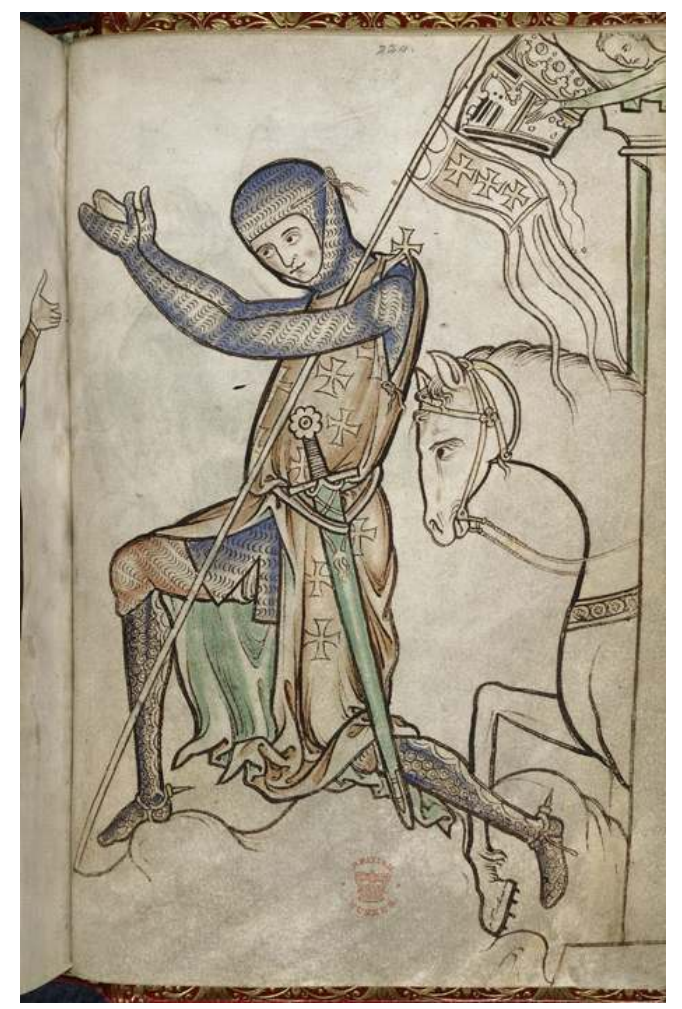

Chevalier agenouillé, page du Psautier de Westminster datée des environs de 1250. Londres, British Library (Royal Ms 2a xxii, fol. 220).

Creative commons. (C) British Library.

Celui-ci est paré de tous les attributs de sa classe : un équipement militaire complet des plus modernes pour l'époque, constitué d'éléments de maille couvrant tout le corps, une épée, sa lance, son heaume, ses éperons, mais également son destrier qui apparaît derrière lui. Ce dernier fait donc partie intégrante de l'iconographie du chevalier. De plus, $\mathrm{du}$ haut de sa monture, celui-ci domine les autres hommes, et affirme physiquement sa supériorité sociale. "Le cheval est donné au chevalier pour signifier la noblesse du courage, pour qu'il soit plus haut monté que les autres hommes » écrit Raymond Lulle dans son Livre de l'ordre de la chevalerie en $1275^{7}$. Mais par extension, le chevalier monté est aussi l'un des symboles du péché d'orgueil dans l'iconographie médiévale, et dans l'enfer représenté sur le tympan de l'abbatiale romane de Sainte-Foy de Conques, celui qui s'en est rendu coupable est violemment jeté à bas de son cheval par les démons (fig. 4). 


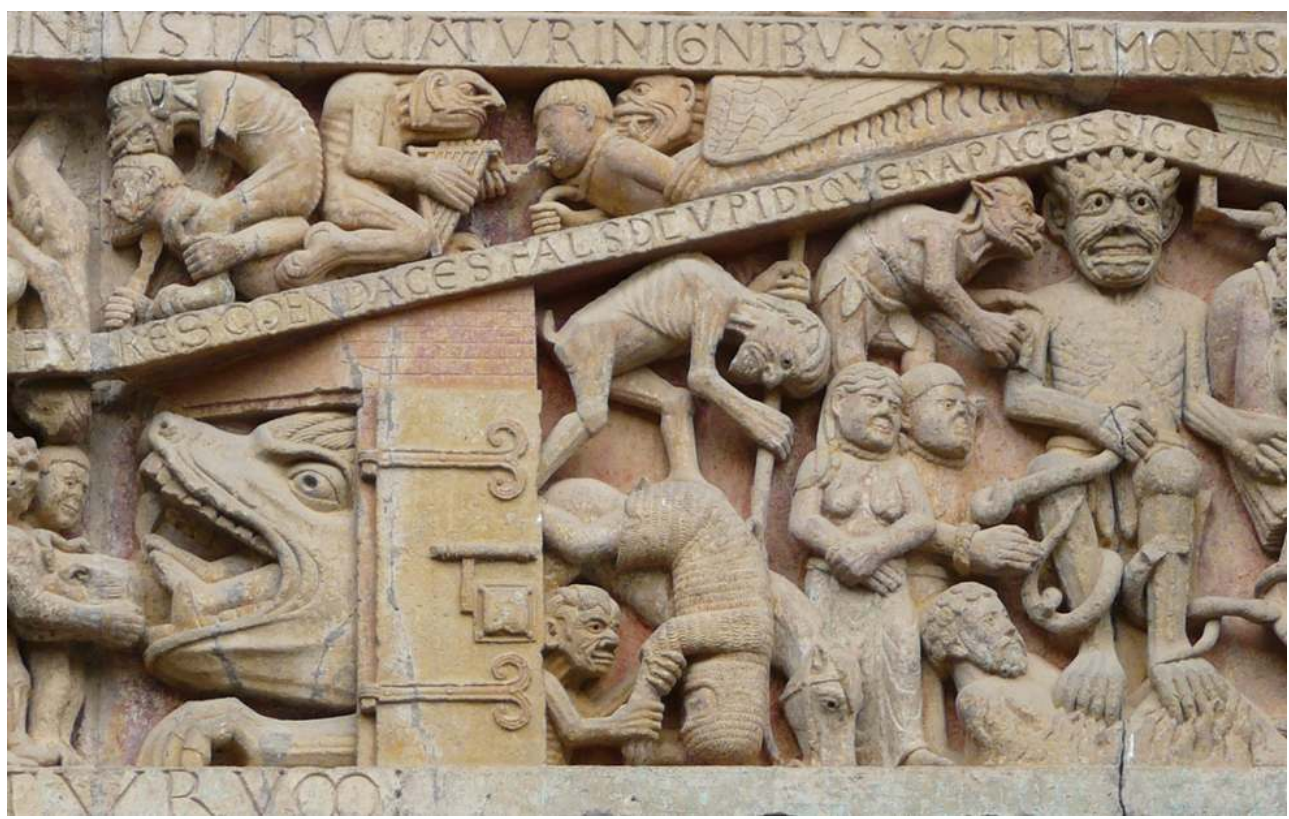

En enfer, le chevalier coupable du péché d'orgueil est jeté à bas de son cheval. Détail d'un Jugement Dernier. Tympan de l'abbatiale Sainte-Foy de Conques, début du XIIe siècle.

(c) Wikimédia.

7 La guerre, et surtout le tournoi, étaient les lieux pour afficher sa noblesse et sa puissance, et la beauté du destrier, mainte fois chantée dans les chansons de geste, était un critère important. Il y avait déjà bien sûr la beauté intrinsèque de l'animal mais aussi tout élément de parure ou d'équipement qui ajoutait à sa splendeur, et par là même, au prestige de son cavalier. Les destriers étaient donc, lorsque les moyens du chevalier le permettaient, volontiers parés de harnachements et de tissus précieux. Les longues housses couvrant l'animal de la tête jusqu'aux pieds, que l'on retrouve sur de nombreuses images médiévales participaient à cet effet. Apparues au milieu du XIII ${ }^{\mathrm{e}}$ siècle, ces housses n'étaient vraisemblablement pas armoriées à l'origine, ce qui pose encore des questions quant à leur fonction première. Protection du harnachement et des bardes de mailles contre les intempéries et le soleil ? Imitation des housses et bardes textiles de certains guerriers orientaux rencontrés durant les croisades ou via l'Espagne ? Dans tous les cas, si celles des chevaux de la Bible de Maciejowski (vers 1245, Pierpont Morgan Library, New York) présentent encore des surfaces unies, dès la seconde moitié du siècle elles vont devenir l'espace privilégié du chevalier pour afficher ses armoiries (fig. 5). 
Figure 5

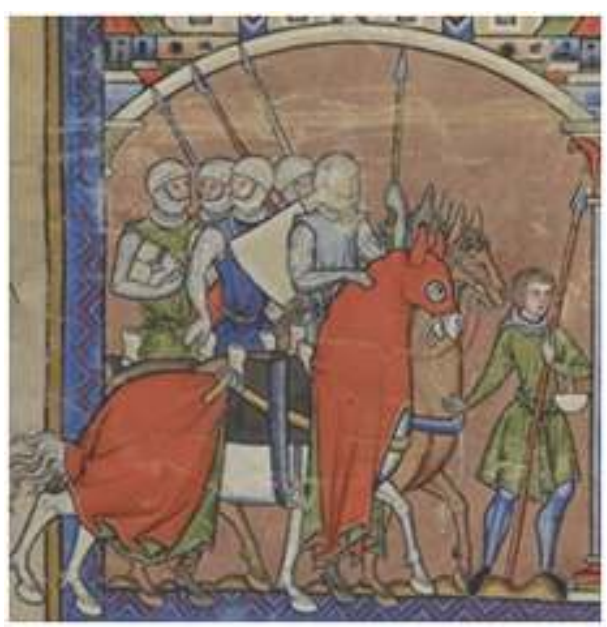

David tuant Shobach, le capitaine de l'armée syrienne. Bible de Maciejowski, vers 1244-1254, détail du folio 2v (scènes de la vie d’Absalom) du manuscrit MS nouv. acq. lat. 2294.

(c) BnF.

8 Ces dernières servaient de moyen de reconnaissance à la guerre et au tournoi, et alors que le bouclier s'était grandement réduit depuis le $\mathrm{xI}^{\mathrm{e}}$ siècle en conséquence des améliorations des défenses de corps en métal, les déployer sur son cheval était le meilleur moyen de se faire reconnaître de loin. De plus, de par leur amplitude, elles offraient aussi peut-être une certaine protection, même légère. Malheureusement ces magnifiques parures ne nous sont plus connues que par l'iconographie, même si certains fragments de tissus armoriés conservés pourraient avoir été à l'origine des housses de cheval. C'est le cas par exemple de la broderie aux léopards d'Angleterre du XIV siècle du musée de Cluny à Paris (fig. 6). 


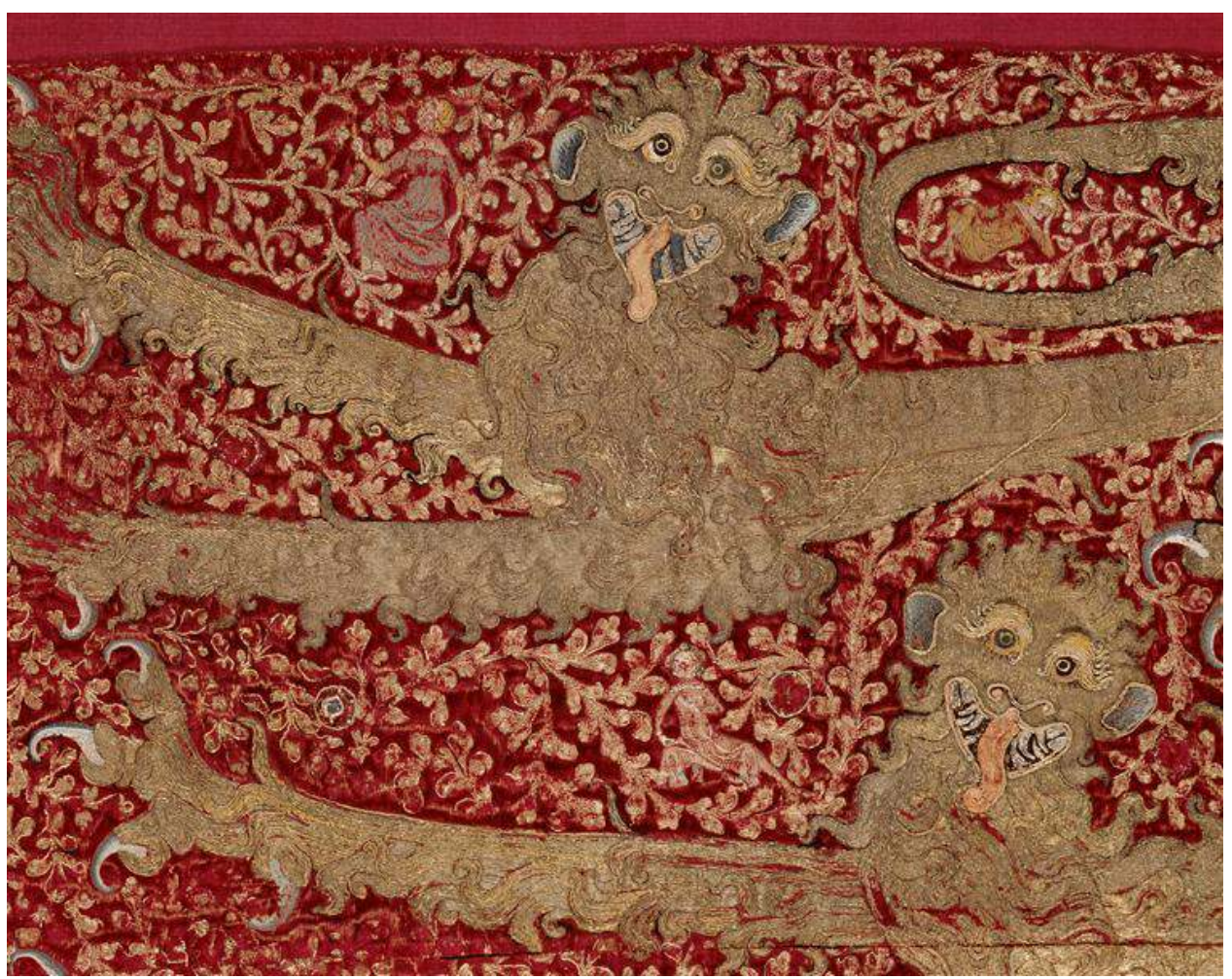

Broderie aux léopards d'Angleterre, peut-être issue d'une housse de cheval ayant appartenu à Édouard III, 1330-1340. Paris, Musée national du Moyen-âge.

(c) RMN-Grand Palais (musée de Cluny - musée national du Moyen-Âge/Franck Raux (cote cliché RMN : 00-011247).

Elle témoigne de la richesse des textiles dont pouvaient être vêtus ces animaux de prestige. En tournoi, les chevaliers abandonnaient parfois leur blason familial au profit de badges et de devises personnelles, louant des vertus morales ou amoureuses. La Relation du pas de Saumur, relatant un tournoi organisé par René d'Anjou en 1446 (peu après 1446, Bibliothèque nationale de Russie, Saint-Pétersbourg, ms. fr. F. p.XIV,4), montre le cheval de ce dernier revêtu d'une housse semée pour la circonstance de fleurs de pensée, son cimier à lambrequin seul permettant aux yeux des spectateurs de l'identifier comme duc d'Anjou. Les cimiers, ces éléments extravagants à signification héraldique, morale ou humoristique placés au sommet des heaumes des tournoyeurs, étaient parfois également fixés sur la tête des chevaux. On le voit par exemple sur l'une des enluminures du Codex Manesse représentant Goesli von Ehnheim lors d'un tournoi (1310-1340, université de Heidelberg) (fig. 7). Lui et son cheval portent sur la tête le même perroquet vert, armes des Ehnheim, faisant ainsi du cheval une sorte de double du chevalier, l'effet étant renforcé ici par le heaume miniature placé sous le cimier du destrier. Faites de matériaux légers, comme le cuir ou le papier mâché, ces structures étaient bien trop fragiles et encombrantes pour être portées à la guerre, et elles survivaient rarement au-delà d'un tournoi, le but étant même souvent d'endommager et de faire tomber le cimier de ses adversaires. 


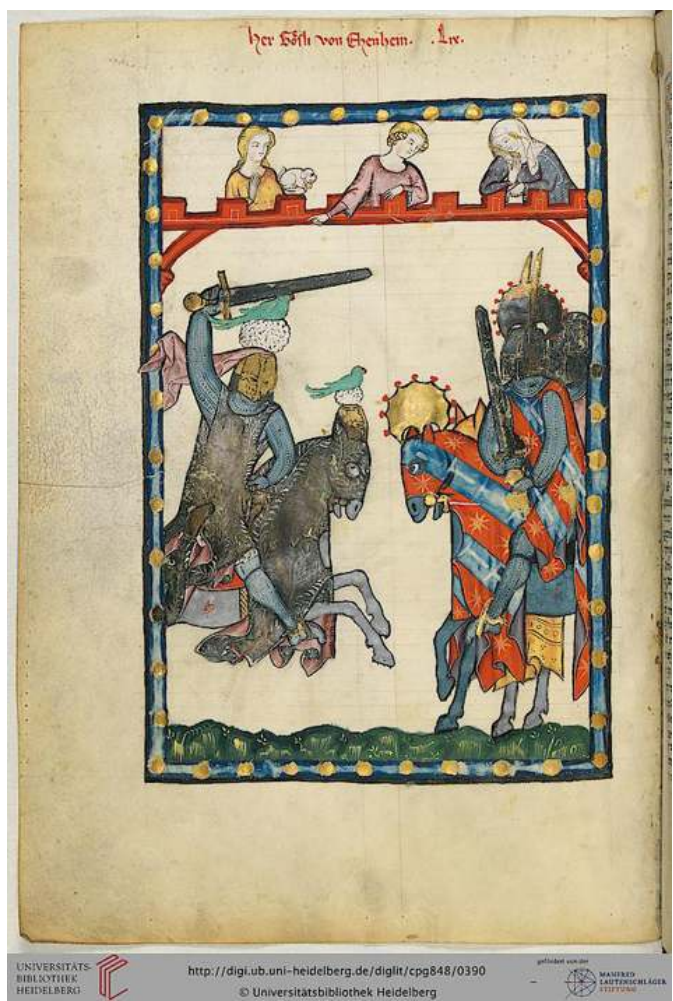

Le chevalier minnesanger Goesli von Ehenheim lors d'un tournoi, Codex Manesse, 1300-1340. Heidelberg, Bibliothèque de l'université (Cod. Pal. Germ. 848, fol. 197v).

(c) Universitätsbibliothek Heidelberg.

Cependant, à la guerre comme au tournoi, cette précieuse monture était très exposée aux blessures et dès le $\mathrm{XII}^{\mathrm{e}}$ siècle, de nombreuses images nous prouvent que sa protection au combat était un enjeu important. Premièrement car l'animal était coûteux, et c'est pour cela que l'on essayait d'épargner ceux des adversaires à la guerre, dans l'espoir de les récupérer, et qu'il était fortement condamnable de blesser volontairement celui d'un concurrent au tournoi. Ensuite et par-dessus tout, avoir son cheval blessé ou tué pendant la bataille était très dangereux pour un chevalier, qui pouvait alors perdre le contrôle d'une monture agonisante, voire devoir combattre à pied, lieu où son équipement lui donnait moins d'avantage. Entre le $\mathrm{XII}^{\mathrm{e}}$ et la fin du $\mathrm{XIV}^{\mathrm{e}}$ siècle la principale forme d'armure pour le cheval, comme pour l'homme d'ailleurs, reste la cotte de mailles. Cette protection, très longue à réaliser et donc coûteuse, surtout pour couvrir l'ensemble du corps d'un cheval, devait être là encore réservée aux plus fortunés des chevaliers. Si sa souplesse ne limite pas les mouvements, cette dernière a cependant l'inconvénient pour l'animal d'être très pesante. Peu à peu, l'armure du cheval s'agrémente d'éléments rigides, en cuir puis en métal, notamment au niveau de la tête et du cou, parties du corps les plus exposées. Ainsi, à partir $d u \mathrm{Xv}^{\mathrm{e}}$ siècle, des bardes entièrement rigides peuvent protéger de manière optimale le corps des chevaux (fig. 8). 




Deux éléments d'une barde de poitrail en cuir bouilli, début du XVIe siècle. New York, Metropolitan Museum of Art.

(C) The Metropolitan Museum of Art.

11 Cependant, les armuriers médiévaux n'ont jamais réussi à concevoir de protection efficace pour les membres des animaux, pourtant très vulnérables aux piétons, sans en restreindre leur mobilité. Si Lorenz Helmschmid (att. 1467-1515/16), grand armurier de Maximilien $\mathrm{I}^{\mathrm{er}} \mathrm{d}^{\prime}$ Autriche, réalisa autour de 1500 des armures articulées complètes pour chevaux, jambes incluses, ces dernières relevaient plus du chef-d'œuvre d'armurerie que de l'équipement véritablement fonctionnel, et ces prototypes restèrent sans suite. De plus, il ne faut pas imaginer les champs de bataille de la fin du Moyen Âge étincelants de chevaux entièrement bardés de fer, vision biaisée par les nombreuses et somptueuses bardes de chevaux conservées dans nos musées. Les documents iconographiques contemporains nous confirment que ces dernières, lourdes et peu stables, étaient visiblement surtout utilisées pour le tournoi et la parade. À la guerre, les destriers armés portaient des bardes similaires, mais réalisées en cuir bouilli peint ou recouvert de riches textiles, en dehors du chanfrein et de la barde de crinière qui pouvaient être en métal (fig. 9, fig. 10). 


\section{Figure 9}

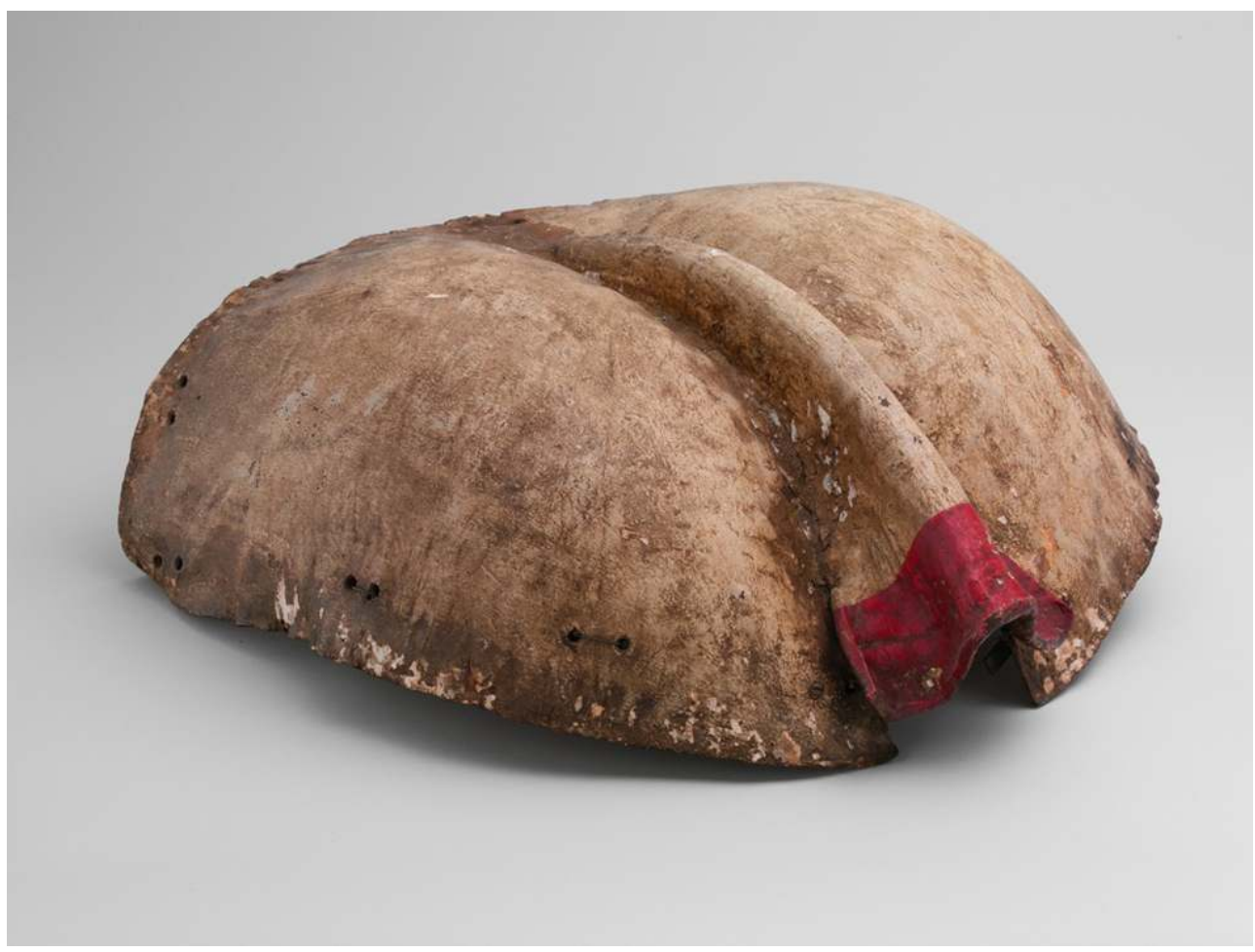

Partie supérieure d'une croupière (barde de croupe) en cuir bouilli, début du XVIe siècle. New York, Metropolitan Museum of Art.

\section{(C) THE METROPOLITAN MUSEUM OF ART.}


Figure 10

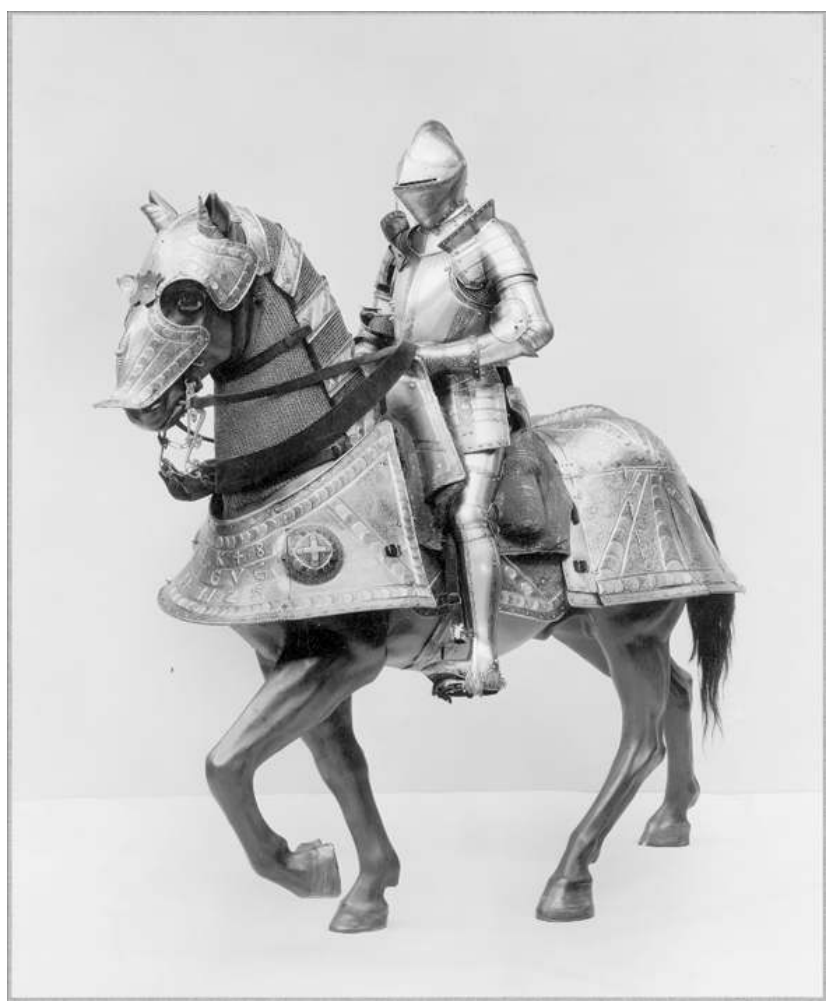

Armure et barde de cheval, Kunst Lochner, 1548. New York, Metropolitan Museum of Art.

(c) THE METROPOLITAN MUSEUM OF ART.

12 Ces dernières étaient un excellent compromis entre solidité et légèreté, étant de plus peu chères et rapides à réaliser. C'est équipé de bardes de ce type que François $\mathrm{I}^{\mathrm{er}}$ et ses compagnons chevaliers combattirent à Marignan, et ainsi qu'ils sont représentés sur le tombeau du roi à Saint-Denis (fig. 11). Bien que très communes à ces époques, elles étaient plus fragiles que celles en métal et d'une durée de vie limitée, et celles qui ont été conservées se comptent sur les doigts d'une main. 
Figure 11

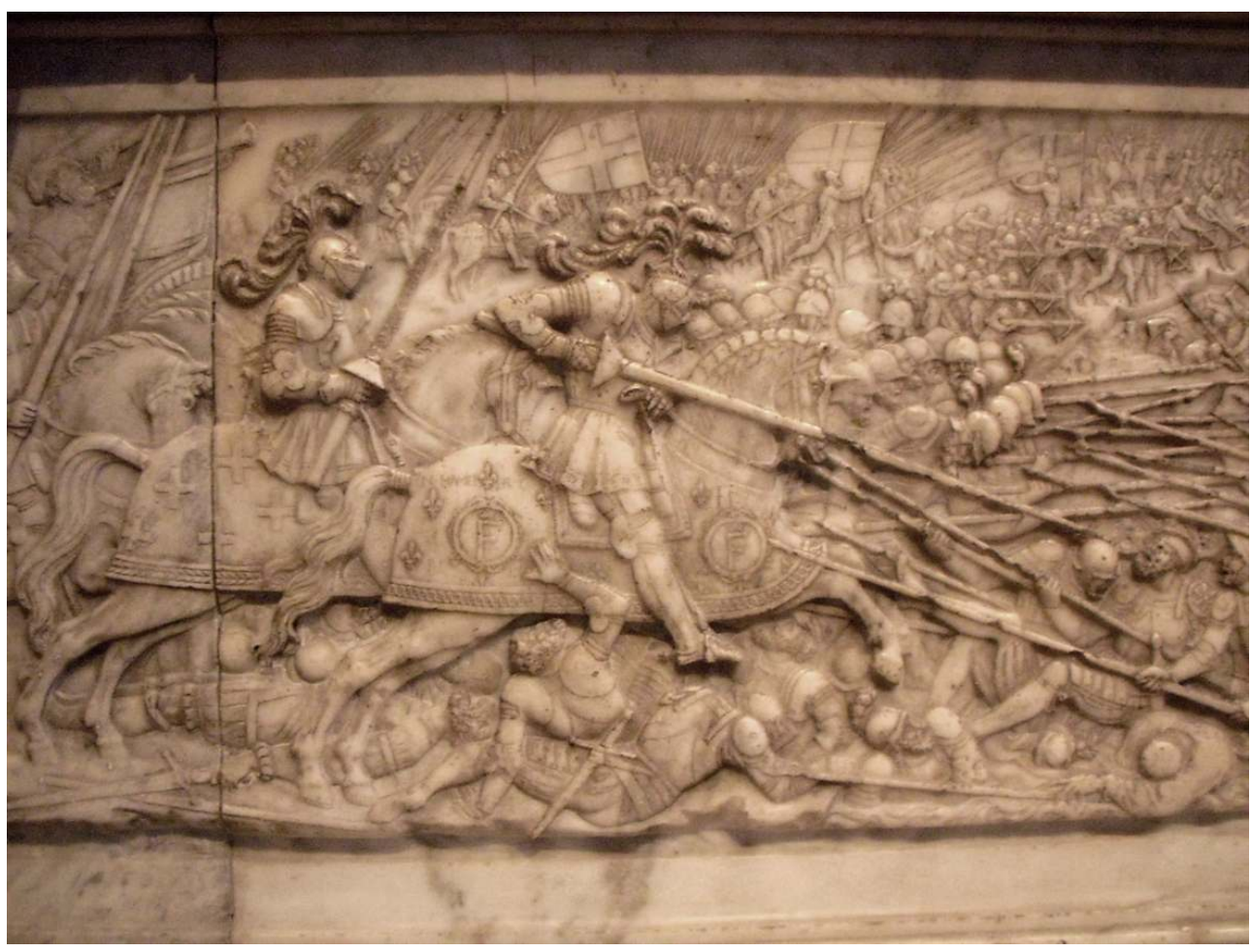

François |er chargeant à cheval à la bataille de Marignan. Pierre Bontemps, relief du soubassement du tombeau de François $\left.\right|^{\text {er }}$ et Claude de France, 1548-1558, basilique Saint-Denis.

Phot. Marina Viallon. (c) Marina Viallon.

Les représentations du cheval de guerre au Moyen Âge sont nombreuses. Elles nous renseignent sur le rôle à la fois technique et surtout symbolique de cet animal au sein de la société chevaleresque, et permettent de compléter d'autres sources plutôt discrètes, voire inexistantes. Cependant, comme dans toute utilisation de sources iconographiques, il faut être prudent et comprendre ce que les artistes ont voulu nous montrer, en recoupant ces informations avec ce que nous connaissons des réalités matérielles de ces époques. Néanmoins, à travers elles, nous pouvons dire du destrier médiéval qu'il était l'un des symboles absolus d'une classe qui justifiait sa domination sociale dans une pratique élitiste et une véritable mise en scène du combat à cheval, de fait bien plus souvent lors de tournois qu'au sein de guerres réelles. Nul doute que ces nombreuses et merveilleuses images sont responsables du fait que le noble chevalier et son fier destrier restent pour nous une image forte de la chevalerie et du Moyen Âge en général.

\section{NOTES}

1. - Marina Viallon, historienne de l'art, est spécialiste en armes et armures anciennes, équipement équestre médiéval et Renaissance. 
2. - GILLMOR, Carol. «Practical Chivalry : The Training of Horses for Tournaments and Warfare». Studies in Medieval and Renaissance History, n.s. 13, 1992, p. 15.

3. - Ibid, p. 9.

4. - Une armure de guerre complète, telle qu'elle apparaît au $\mathrm{xv}^{\mathrm{e}}$ siècle, pesait une vingtaine de kilos environ : elle était donc loin d'être un poids particulièrement handicapant pour le cheval et son cavalier. Les armures spécialisées pour le tournoi, en revanche, comportant de nombreux renforts, pouvaient être jusqu'à deux fois plus lourdes, mais l'enjeu était alors sportif et elles étaient portées très peu de temps.

5. - La taille minimale d'un cheval de pure race espagnole aujourd'hui est d'1,50 m pour les femelles et 1,52 m pour les mâles.

6. - Archéologie du cheval. Des origines à la période moderne en France. Paris : Errance, 2002, p. 46-49.

7. - LULLE, Raymond. Le Livre de l'ordre de la chevalerie. Introduction, traduction et notes par Bruno Hapel. Paris : G. Trédaniel, 1990, p. 58.

\section{RÉSUMÉS}

Le destrier était le cheval de guerre et de tournoi du chevalier. On ne saurait cependant relativement que peu de choses sur ce cheval au Moyen Âge, sans les très nombreuses représentations, qui, $\mathrm{du} \mathrm{XI}^{\mathrm{e}}$ au $\mathrm{XVI}^{\mathrm{e}}$ siècle, en font un élément omniprésent de l'art militaire médiéval. Ces images nous présentent un cheval dont la rapidité et la force étaient nécessaires à la pratique d'un combat exclusivement chevaleresque développé à la fin $\mathrm{du} \mathrm{XI}^{\mathrm{e}}$ siècle : la charge à la lance couchée. Mais le destrier fait aussi la fierté de celui qui le monte. À travers les représentations de cette monture de grand prix, à l'équipement souvent lui-même coûteux, l'imagerie médiévale met aussi en valeur la noblesse et la richesse de son cavalier.

Trusty steeds, images of warhorses in the Middle ages. The destrier was the knight's war and tournament horse. Yet we would know almost nothing about this horse in the Middle Ages without the numerous representations that make this type of animal an omnipresent figure in medieval art from the eleventh to the sixteenth century. These images show us a horse of which the speed and the strength were necessary for the practice of a very knightly kind of combat: the charge with the couched lance. Then the destrier must do his rider proud. Through the representations of this luxurious horse, often wearing costly trappings, medieval imagery highlights the nobility and the wealth of its rider.

\section{INDEX}

Mots-clés : cheval, chevalerie, tournois, guerre, Moyen Âge, enluminure, armures, arts

Keywords : horse, chivalry, tournament, war, Middle Ages, illuminations, armour, arts 


\section{AUTEUR}

\section{MARINA VIALLON}

historienne de l'art marina.viallon@yahoo.fr 